\title{
ARTICLE OPEN \\ Generalized Ramsey interferometry explored with a single nuclear spin qudit
}

\author{
Clément Godfrin ${ }^{1}$, Rafik Ballou $\mathbb{D}^{1}$, Edgar Bonet ${ }^{1}$, Mario Ruben $\mathbb{D}^{2,3}$, Svetlana Klyatskaya ${ }^{2}$, Wolfgang Wernsdorfer ${ }^{1,2,4}$ and \\ Franck Balestro ${ }^{1,5}$
}

Qudits, with their state space of dimension $d>2$, open fascinating experimental prospects. The quantum properties of their states provide new potentialities for quantum information, quantum contextuality, expressions of geometric phases, facets of quantum entanglement and many other foundational aspects of the quantum world that are unapproachable via qubits. Here, we have experimentally investigated the quantum dynamics of a qudit $(d=4)$ that consists of a single $3 / 2$ nuclear spin embedded in a molecular magnet transistor geometry, coherently driven by a microwave electric field. In order to demonstrate the potentialities of molecular magnets for quantum technologies, we implemented three protocols based on a generalization of the Ramsey interferometry to a multilevel system. First, the Ramsey interference is used to measure the accumulation of geometric phases. Then, two distinct transitions of the nuclear spin are addressed to measure the phase of an iSWAP quantum gate. Finally, through a succession of two Hadamard gates, the coherence time of a 3-state superposition is measured.

npj Quantum Information (2018)4:53; doi:10.1038/s41534-018-0101-3

\section{INTRODUCTION}

A universal quantum computer requires the coherent control of large Hilbert spaces, ${ }^{1-3}$ which makes its achievement an ambitious technical challenge. The traditional approach is to build a largescale quantum coherent architecture with two-states quantum devices (qubits) into which information is encoded and treated using state populations and phases. An alternative path to overcome the scalability obstruction is to make use of $d$-states devices $^{4,5}$ (qudits) as basic building bloc. Indeed, several proposals and experiments recently demonstrated that multilevel quantum systems can be of great relevance to the field of quantum information processing ${ }^{6-17}$ provided that there is a long coherent control of the system's phase ${ }^{18,19}$ and possibility of implementing quantum error protocols. ${ }^{20}$ In general, interferometric circuits are used to access information of a phase: an initial state is subjected to two paths before merging into a final state. Depending on the difference between the phases accumulated on the two different paths, the state will recombine in a constructive or destructive interference. The information on the phase difference between the two paths can be deduced via a population measurement. Furthermore, the phase coherence ${ }^{21}$ can be assessed by the contrast of the interference fringes. The archetype interference experiment based on Young slits proved the wave/particle character of light ${ }^{22}$ and single atoms. ${ }^{23}$ Nowadays, Ramsey interferometry is widely used to characterize the coherence time of qubits. Here, we employ a generalization of Ramsey interferometry to a single nuclear spin qudit, which we have implemented in three different protocols. The first protocol (single-transition Ramsey interferometry) allows a phase measurement of quantum dynamics that preserves the state population.
We use it to determine the geometric phase accumulated by quantum states driven along closed paths in their state spaces. The second protocol (double-transition Ramsey interferometry) is more general by demonstrating our ability to measure a phase of a quantum evolution, even if the state populations have been modified. We apply it to the iSWAP quantum gate. The third protocol (Hadamard-Ramsey interferometry) is suited in essence to measure phases of multilevel-state superposition. We have illustrated it here by measuring the coherence time of a threestate superposition. All these protocols can be generalized for the case of any $d$-state system and they are universal. They have been illustrated by measurements performed on a single $3 / 2$ nuclear spin located in a molecular magnet. ${ }^{12,24,25}$

\section{RESULTS}

Molecular spins have attracted the interest of different communities in the last few decades because of the possibility to tailor their magnetic and quantum properties at a synthetic level. They can be deposited and positioned on different substrates, and the bottom-up massive production of identical molecular units is relatively cheap. Key experiments ${ }^{26}$ have shown that the spin coherent lifetime and entanglement can be tailored by suitable molecular engineering thus showing potentials for quantum technologies. In this work, we exploit a bis-phthalocyanine terbium (III) single-molecule magnet $\left(\mathrm{TbPc}_{2}\right)$, embedded in a single-electron transistor (Fig. 1a). The core of the molecule is a $\mathrm{Tb}^{3+}$ ion with an electronic angular momentum $J=6$ and a nuclear spin $I=3 / 2$. Due to the ligand field of the phthalocyanines, the electronic angular momentum behaves like a \pm 6 Ising

\footnotetext{
${ }^{1}$ Univ. Grenoble Alpes, CNRS, Grenoble INP, Institut Néel, 38000 Grenoble, France; ${ }^{2}$ Institute of Nanotechnology, Karlsruhe Institute of Technology, 76344 EggensteinLeopoldshafen, Germany; ${ }^{3}$ Institute de Physique et Chimie de Matériaux (IPCMS), Université Strasbourg, 23, rue du Loess, BP 43, 67034 Strasbourg cedex 2, France; ${ }^{4}$ Physikalisches

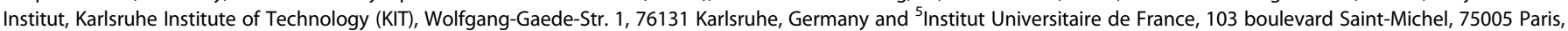
France

Correspondence: Wolfgang Wernsdorfer (wolfgang.wernsdorfer@neel.cnrs.fr) or Franck Balestro (franck.balestro@neel.cnrs.fr)
}

Received: 5 February 2018 Revised: 13 September 2018 Accepted: 27 September 2018

Published online: 24 October 2018 

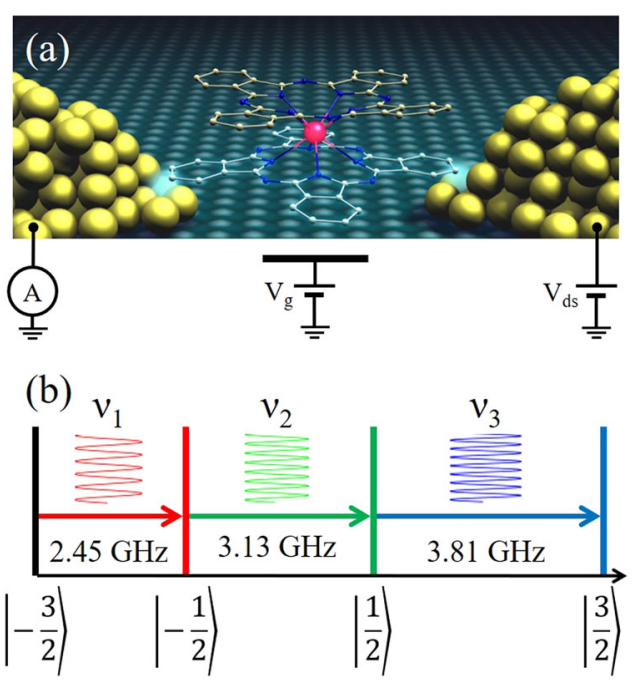

Fig. 1 Qudit Scheme. a The $\mathrm{TbPc}_{2}$ molecular magnet is embedded in a transistor. Transport measurement off the transistor under magnetic field enables the read-out of the $3 / 2$ nuclear spin carried out by the $\mathrm{Tb}^{3+}$ ion (inspired from refs. ${ }^{25,28}$ ). b Energy diagram of the four nuclear spin states. The quadruple component in the hyperfine coupling enables an independent manipulation of each transition

spin. Its axis is used as a quantization axis $z$ for the nuclear spin. The hyperfine interaction between the electronic and the nuclear spin consists of a dipolar term $\mathrm{A}=0.52 \mathrm{GHz}$ and a quadrupolar term $P=0.34 \mathrm{GHz} .{ }^{27}$ This specific hyperfine coupling gives rise to a four-level quantum system with three distinct resonance frequencies $v_{1} \approx 2.45 \mathrm{GHz}, v_{2} \approx 3.13 \mathrm{GHz}$ and $v_{3} \approx 3.81 \mathrm{GHz}^{12}$ (Fig. 1b). This quadrupolar term is two orders of magnitude larger than the resonance widths, thus avoiding any cross-talk in between different transitions. An exchange coupling in between the $\mathrm{Tb}^{3+}$ ion electronic spin and the spin carried out by the $\mathrm{Pc}$ read-out quantum dot induces an electronic spin dependence of the conductance through the read-out quantum dot, allowing a direct read-out of this electronic spin via transport measurement. ${ }^{28}$ Furthermore, this electronic spin has a finite probability to tunnel from one state to the other through quantum tunnelling of magnetization (QTM) governed by the Landau-Zener process. As a result of the hyperfine coupling, QTM can occur at four different magnetic fields, corresponding to the four nuclear spin states. Therefore, the magnetic field values at conductance jumps read out the nuclear spin states. ${ }^{24}$ Finally, an antenna in the vicinity of the transistor allows a coherent manipulation of the nuclear spin transitions using only the electric field. ${ }^{12,25}$ The device is cooled down to $40 \mathrm{mK}$ by means of a dilution refrigerator and subjected to a magnetic field created via a 3D-vector magnet. The probability of being in a given nuclear spin state, knowing the initial one, is then measured using the following protocol: (i) Preparation: magnetic field swept from $+60 \mathrm{mT}$ to $-60 \mathrm{mT}$ to read out the initial nuclear spin state. (ii) Evolution: application of electric microwave pulses at a constant external magnetic field. (iii) Reading-out: magnetic field swept back from $-60 \mathrm{mT}$ to $+60 \mathrm{mT}$ to read out the final nuclear spin state. The entire sequence is rejected when no QTM transition is detected. After repeating the procedure 500 times, we yield the probability $P_{p, q}$ of the state $|q\rangle$ knowing that the initial state is $|p\rangle$ for a given pulse sequence $P_{p, q}=N_{p, q} / \sum_{n} N_{p, n}$, where $N_{p, q}$ counts the number of events for which the QTM reveals a state $|p\rangle$ before the pulse sequence and $|q\rangle$ after. The repetition of this sequence for different pulse lengths gives access to the dynamics of each state under the influence of the microwave pulse.
The microscopic mechanism by which the nuclear spin can be controlled with an electric field relies on the dependence of the hyperfine interactions on the electric field, which is amplified by the significant strengthening of the Stark interactions by the oddparity contribution to the ligand field of the phthalocyanines on the $\mathrm{Tb}^{3+}$ ion causing a minor mixing of electronic states of opposite parity. ${ }^{12,25}$ Based on this hyperfine Stark effect, a timedependent electric field behaves with respect to the nuclear spin I in an analogue way to a magnetic field in rotation in the $(x, y)$ plane perpendicular to the electronic spin axis $z$ with the same pulsations and phase shifts as the electric ones. Since the nuclear spin transverse operators $\left(\boldsymbol{I}_{x}, \boldsymbol{I}_{y}\right)$ are connecting each state $|M\rangle$ to the states $|M \pm 1\rangle$ the coherent control of the nuclear spin can be achieved through the three Rabi oscillations $|-3 / 2\rangle \leftrightarrow|-1 / 2\rangle, \mid-1 /$ $2\rangle \leftrightarrow|+1 / 2\rangle$ and $|+1 / 2\rangle \leftrightarrow|+3 / 2\rangle$, each taking place for a distinct pulsation (Fig. 1b). Whenever the manipulation of states is performed through a succession of monochromatic pulses, the dynamics can be accounted for by making use of the Bloch-sphere representation. The evolution after a time $\tau$ of an initial nuclear spin state $|\Psi(0)\rangle$ subjected to a monochromatic pulse with the pulsation $\omega_{p q}$ of a $|p\rangle \leftrightarrow|q\rangle$ Rabi oscillation and a phase shift $\varphi$ can be described in the rotating frame by $\left|\psi\left(\omega_{p q} \tau\right)\right\rangle=\mathrm{R}_{\varphi}^{|p\rangle,|q\rangle}\left(\omega_{p q} \tau\right)|\psi(0)\rangle$, with:

$\mathrm{R}_{\varphi}^{|p\rangle,|q\rangle}(\theta)=\exp \left[i \theta\left(\cos \varphi \boldsymbol{\sigma}_{x}^{|p\rangle,|q\rangle}+\sin \varphi \boldsymbol{\sigma}_{y}^{|p\rangle,|q\rangle}\right) / 2\right]$

where $\left(\sigma_{x}^{|\mathbf{p}\rangle,|q\rangle}, \sigma_{y}^{|\mathfrak{p}\rangle,|q\rangle}\right)$ operates on the space of states generated by $|p\rangle$ and $|q\rangle$ in a similar way to transverse Pauli operators in a spin- $1 / 2$ state space and cancels every state of the supplementary space. $R_{\varphi}^{|p\rangle,|q\rangle}(\theta)$ corresponds to a rotation of the angle $\theta$ around the axis at the angle $\varphi$ from the $x$ axis in the $(x, y)$-plane on the Bloch sphere associated with the $|p\rangle \leftrightarrow|q\rangle$ Rabi oscillation (Fig. 2a). In the case where the manipulation of states is performed through a polychromatic pulse, the dynamics can again be accounted for intuitively with rotations in a spin-(N/2) state space for particular ratios of the amplitudes of the polychromatic pulse's $\mathrm{N}$ chromatic components that can be experimentally calibrated (Fig. 2b). Then a transformation is again denoted $\mathrm{R}_{\varphi}^{|\mathrm{p}\rangle,|\mathrm{q}\rangle}(\theta)$ where the states $|p\rangle$ and $|q\rangle$ represent the extreme states of either a triplet $(N=2)$ or a quadruplet $(N=3)$. Notice that Eq. (1) is only valid for resonant monochromatic pulses and for resonant polychromatic pulses that have multiple of $\pi$ duration.

\section{Single-transition Ramsey interferometry: geometric phase}

The phase of a state at any instant in a given protocol can be measured by generalizing the method of Ramsey interferometry as follows. Given a state $|p\rangle$, the two paths of an interferometer are built by creating a quantum superposition with the partner state $|q\rangle$ of a $|p\rangle \leftrightarrow|q\rangle$ Rabi oscillation. This is merely achieved by applying a $\pi / 2$ pulse of pulsation $\omega_{p q}$ during the time $t=(\pi / 2) / \omega_{p q}$. The states $|p\rangle$ and $|q\rangle$ can then be manipulated separately by microwave pulses of distinct pulsations feeding them with independent phases $\varphi_{\mathrm{p}}$ and $\varphi_{\mathrm{q}}$. By applying the same $\pi / 2$ pulse once again, the two arms of the interferometer are merged back leading to a final state whose probability to be in the state $|p\rangle$ (resp. $|q\rangle)$ is given by $\cos ^{2}\left[\frac{\varphi_{p}-\varphi_{q}}{2}\right]\left(\right.$ resp. $\left.\sin ^{2} \frac{\varphi_{p}-\varphi_{q}}{2}\right)$ which reveals the difference in the phases separately accumulated by the two states from the time at which the first $\pi / 2$ pulse was applied up to the time at which the second $\pi / 2$ pulse was started. The computation of these probabilities is shown in detail in the supplementary information. It is important to emphasize that the population of the states $|p\rangle$ and $|q\rangle$ must be unaffected by the transformation that creates the phase difference in between the two paths. The motion of a quantum state on a closed path, which results in the accumulation of a geometric phase, is ideally suited to this protocol. Geometric phases were first discovered by $\mathrm{S}$. 


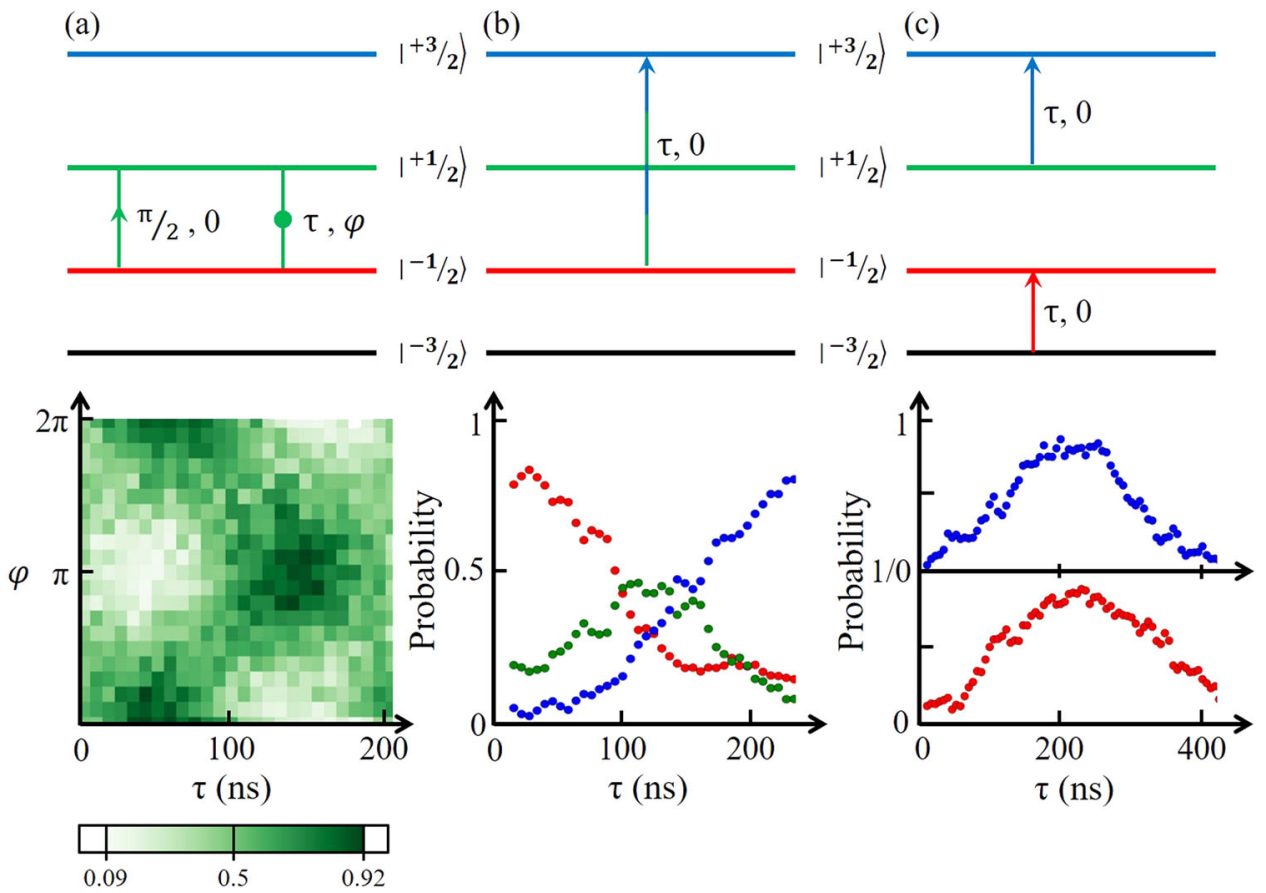

Fig. 2 Qubits coherent manipulation. a A $\pi / 2$ pulse with zero phase projects the state onto the equatorial plane of the Bloch sphere. This first pulse is followed by a second one of duration $\tau$ with a phase $\varphi$. The latter selects the rotation axis at the angle $\varphi$ from the $x$-axis. The experimental measurement of the probability $\boldsymbol{P}_{\mid-1 / 2\}, \mid 1 / 2\}}$ as a function of $\tau$ and $\varphi$ illustrates this dynamic. $\mathbf{b}$ A pulse comprising the frequency of the $2 \mathrm{nd}$ and the $3 \mathrm{rd}$ transition is sent during a time $\tau$. This pulse provides a population inversion in between the red and the blue state for a duration of $230 \mathrm{~ns}$ as observed in the evolution of each probabilities $\boldsymbol{P}_{|-1 / 2\rangle, \boldsymbol{q}}$ as a function of $\tau$. It occurs through the intermediary of the green state. c A pulse comprising the frequency of the 1 st and the 3rd transition is simultaneously sent during a time $\tau$. Probabilities $\left.\boldsymbol{P}_{\mid-3 / 2}, 1-1 / 2\right\rangle$ and $\boldsymbol{P}_{|1 / 2\rangle,|3 / 2\rangle}$ of the red and the blue states starting from the black and the green ones, respectively, is measured as the function of the pulse duration. Populations of these two states are reversed for pulse duration of $210 \mathrm{~ns}$

Pancharatnam ${ }^{29}$ through polarization manipulation in classical optics. Later, M.V. Berry ${ }^{30}$ found geometric phases by analysis of adiabatic cyclic quantum dynamics, thereafter interpreted with a holonomy element in a line bundle, ${ }^{31}$ and finally discussed in its full generality in the context of non-abelian spectral bundles ${ }^{16}$ and for non-cyclic and non-unitary evolutions. ${ }^{32}$ Its global nature makes it less prone to zero average noise sources and fluctuations, thus making it more robust to errors. ${ }^{33-35}$ It was explored in various experiments including molecular magnets ${ }^{36}$ and superconductnig circuits. ${ }^{37,38}$ In order to produce a geometric phase in the nuclear spin qudit, we use a sequence of two consecutive $\pi$ pulses of phase $\varphi$ and $\varphi+\Theta$ associated with a $|p\rangle \leftrightarrow|q\rangle$ transition, which corresponds to a closed path on a sphere composed of two meridian arcs shifted by an angle $\Theta$ and joining the north pole to the south pole. The geometric phase $\gamma_{G}$ is given through the solid angle of the surface sustained by the closed path $\gamma_{G}=|p-q| \Theta$ (see supplementary information). Note that the initial phase $\varphi$ is irrelevant and can be arbitrarily set to zero. In a first experiment we applied the following pulse sequence:

$$
U_{1}=\mathbf{R}_{0}^{|-3 / 2\rangle,|-1 / 2\rangle}(\boldsymbol{\pi} / 2) \cdot \mathbf{R}_{\Theta}^{|-1 / 2\rangle,|1 / 2\rangle}(\pi) \cdot \mathbf{R}_{0}^{|-1 / 2\rangle,|1 / 2\rangle}(\pi) \cdot \mathbf{R}_{0}^{|-3 / 2\rangle,|-1 / 2\rangle}(\boldsymbol{\pi} / 2)
$$

The first and last factors stand for the Ramsey interferometry. The geometric phase here is acquired by the state $|q\rangle=|-1 / 2\rangle$ when moving in the space of states associated with the transition | $q\rangle=|-1 / 2\rangle \leftrightarrow|r\rangle=|1 / 2\rangle$ isomorphic to a spin $|1 / 2\rangle$ state space. It is therefore equal to $\gamma_{G}=|q-r| \Theta=\Theta$. The $q$ arm of the interferometer is fed with the phase $\varphi_{q}=\varphi_{G}$ whereas the $p$ arm remains unfed. It follows that $\varphi_{p}-\varphi_{q}=\Theta$ as observed experimentally and shown in Fig. 3a.

In a second experiment, the geometric phase was accumulated by the state $|q\rangle=|-1 / 2\rangle$ when moving in the space of states associated with $|-1 / 2\rangle \leftrightarrow|3 / 2\rangle$ transition isomorphic to a spin-1 state space:

$$
U_{2}=\mathbf{R}_{0}^{|-3 / 2\rangle,|-1 / 2\rangle}(\boldsymbol{\pi} / 2) \cdot R_{\Theta}^{|-1 / 2\rangle,|3 / 2\rangle}(\pi) \cdot R_{0}^{|-1 / 2\rangle,|3 / 2\rangle}(\pi) \cdot \mathbf{R}_{0}^{|-3 / 2\rangle,|-1 / 2\rangle}(\boldsymbol{\pi} / 2)
$$

The $q$ arm of the interferometer is now fed with the phase $\varphi_{q}=$ $\gamma_{G}=|q-r| \Theta=2 \Theta$, whereas the $p$ arm remains unfed. It follows that $\varphi_{p}-\varphi_{q}=2 \Theta$ as once again observed experimentally and shown in Fig. $3 b$.

In a third and last experiment, we aimed at measuring an interference pattern in between two geometric phases. For this purpose, we applied the pulse sequence:

$U_{1}=\mathbf{R}_{0}^{|-1 / 2\rangle,|1 / 2\rangle}(\boldsymbol{\pi} / 2) \cdot V_{\Theta_{1}} \cdot V_{\Theta_{3}} \cdot \mathbf{R}_{0}^{|-1 / 2\rangle,|1 / 2\rangle}(\boldsymbol{\pi} / 2)$

with

$$
\mathrm{V}_{\Theta_{1}}=\mathrm{R}_{\Theta_{1}}^{|-1 / 2\rangle,|-3 / 2\rangle}(\pi) \cdot \mathrm{R}_{0}^{|-1 / 2\rangle,|-3 / 2\rangle}(\pi)
$$

$\mathrm{V}_{\Theta_{3}}=R_{\Theta_{3}}^{|1 / 2\rangle,|3 / 2\rangle}(\pi) \cdot R_{0}^{|1 / 2\rangle,|3 / 2\rangle}(\pi)$. Unlike previous protocols, here the initial state is $|-1 / 2\rangle$. The $\pi$ pulses on the first and on the third transition must be applied simultaneously and with the same duration as presented in Fig. 2c. The two arms $|p\rangle=|-1 / 2\rangle$ and $|q\rangle$ $=|1 / 2\rangle$ of the interferometer are now fed with a geometric phase, more precisely $\varphi_{p}=\Theta_{1}$ and $\varphi_{q}=\Theta_{3}$ from motions along closed paths in the state spaces associated with the transitions $|-1 / 2\rangle \leftrightarrow \mid$ $-3 / 2\rangle$ and $|1 / 2\rangle \leftrightarrow|3 / 2\rangle$, respectively. The interference pattern in between the two geometric phases is achieved in the second Ramsey $\pi / 2$ pulse as displayed in Fig. $3 c$, where it is seen through the probability of being in the state $|q\rangle$.

Double-transition Ramsey interferometry: quantum gate Quantum operations on composite qubits can always be decomposed into a set of one-qubit gates and two-qubits gates. It proves of utmost interest to try implementing the latter on a non-composite system, such as a single qudit that can thus 

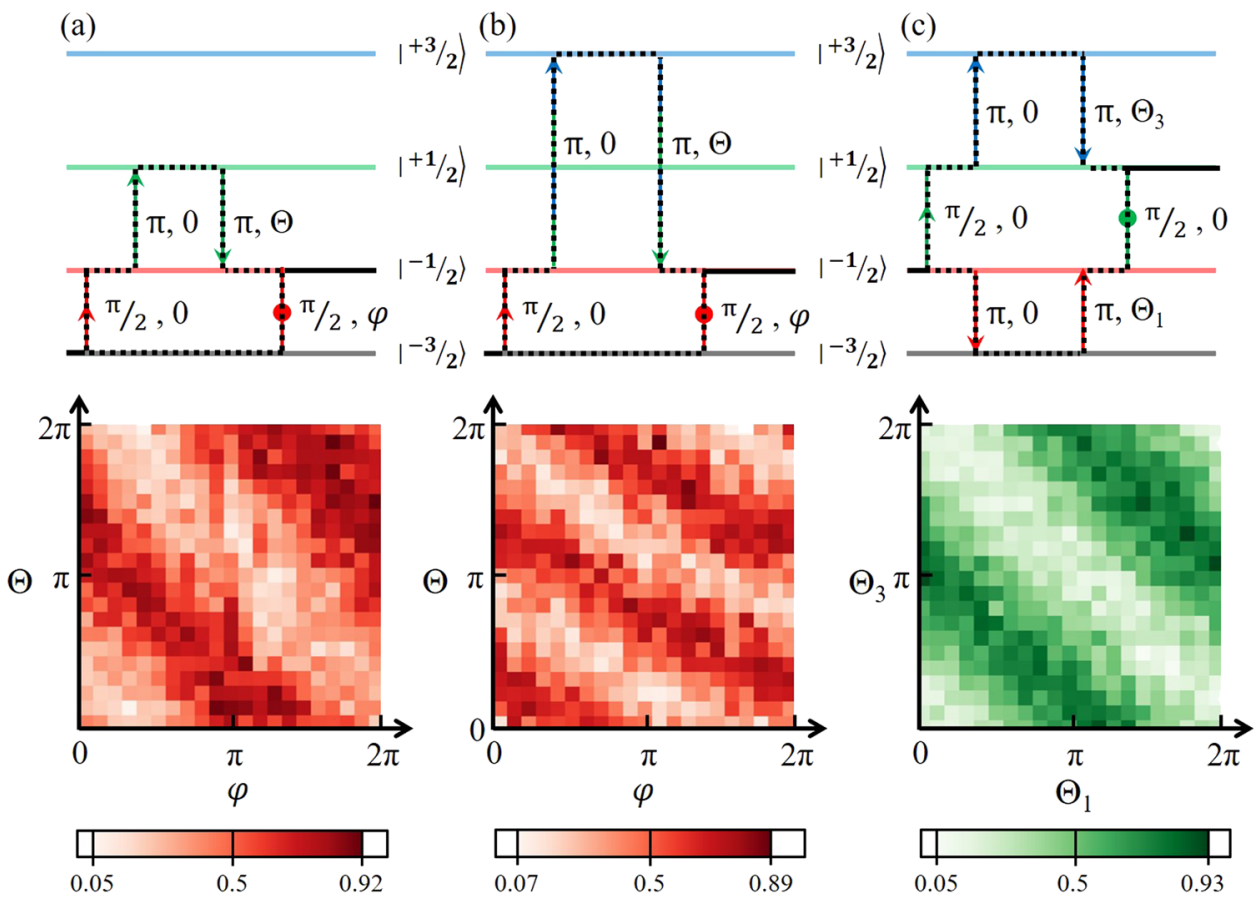

Fig. 3 Geometric phase. a The system is initialized in the black state. A $\pi / 2$ pulse on the first transition with zero phase creates a coherent superposition of the black and the red state. While no pulse is applied to the black state, a sequence of two $\pi$ pulses is sent on the 2 nd transition. The phase difference between these two pulses being $\Theta$, a geometric phase equal to $\Theta$ is accumulated. Finally, a second $\pi / 2$ pulse is sent on the first transition with a phase $\varphi$. This creates $\cos ^{2} \frac{\Theta+\varphi}{2}$ interference between the black and the red states, revealed by the probability $P_{|-3 / 2\rangle,|-1 / 2\rangle}$ map as the function of $\varphi$ and $\Theta$. b The system is initialized in the black state. A $\pi / 2$ pulse on the first transition with zero phase creates a coherent superposition of the black and the red state. While no pulse is applied to the black state, a sequence of two $\pi$ pulses is sent on the 2nd and the 3rd transition, as shown in Fig. 2b. The phase difference between these two pulses being $\Theta$, a geometric phase equal to $2 \Theta$ is accumulated. Finally, a second $\pi / 2$ pulse is sent on the first transition with a phase $\varphi$. It $\operatorname{creates} \cos ^{2} \frac{2 \theta+\varphi}{2}$ interference between the black and the red states, revealed by the probability $P_{|-3 / 2\rangle,|-1 / 2\rangle}$ map as the function of $\varphi$ and $\Theta$. c The system is initialized in the red state. A $\pi / 2$ pulse on the second transition with a zero phase creates a coherent superposition of the red and the green states. Simultaneously, a sequence of two $\pi$ pulses is sent on the 1st and the 3rd transition, as shown in Fig. 2c The phase difference between these two pulses being $\Theta_{1}$ and $\Theta_{3}$, respectively. As a consequence geometric phases equal to $\Theta_{1}$ and $\Theta_{3}$ are accumulated on the red and on the green states, respectively. Finally, a second $\pi / 2$ pulse on the first transition with a zero phase creates $\cos ^{2} \frac{\Theta_{1}+\Theta_{3}}{2}$ interference between the red and the green states, revealed by the probability $P_{|-1 / 2\rangle,|1 / 2\rangle}$ map as the function of $\Theta_{1}$ and $\Theta_{3}$. This interference pattern involves geometric phases only

provide useful resources for quantum technologies. ${ }^{39}$ For this purpose, we have investigated how a qudit $(\mathrm{d}=4)$ enables an iSWAP gate. According to the following one-to-one map

$$
\begin{aligned}
& \left.|-3 / 2\rangle \leftrightarrow\left|0_{A}\right\rangle \otimes\left|0_{B}\right\rangle,|-1 / 2\rangle \leftrightarrow\left|0_{A}\right\rangle \otimes\left|1_{B},\right| 1 / 2\right\rangle \leftrightarrow\left|1_{A}\right\rangle \otimes\left|0_{B}\right\rangle,|3 / 2\rangle \\
& \quad \leftrightarrow\left|1_{A}\right\rangle \otimes\left|1_{B}\right\rangle
\end{aligned}
$$

between the basis states of a qudit $(\mathrm{d}=4)$ and those of two entangled qubits, one might expect from a quantum iSWAP gate that does not modify the states $|-3 / 2\rangle$ and $|3 / 2\rangle$ while it switches the states $|-1 / 2\rangle$ and $|1 / 2\rangle$ and feeds them with an additional $\pi / 2$ phase without modifying the states $|-3 / 2\rangle$ and $|3 / 2\rangle$ :

$$
U_{\text {iSWAP }}=\left[\begin{array}{llll}
1 & 0 & 0 & 0 \\
0 & 0 & i & 0 \\
0 & i & 0 & 0 \\
0 & 0 & 0 & 1
\end{array}\right]=\mathrm{R}_{0}^{|-1 / 2\rangle,|1 / 2\rangle}(3 \pi)
$$

As displayed in Eq. (6), an iSWAP quantum gate is implemented in a qudit through merely a resonant $3 \pi$ pulse applied on the 2 nd transition of the 4-level spectrum (Fig. 4a). However, the quantum gate affects both the phase and population of the states making it impossible to apply the previous Ramsey interferometry for the measurement of its phase. It is like attempting to measure the phase of an event that simultaneously acts on both arms of the interferometer. In order to circumvent this difficulty, we propose to make use of a third arm in which we isolate one path that is later used to interfere with the path modified by the implementation of the quantum gate. This is a more general Ramsey interferometry where the two $\pi / 2$ pulses are not applied on the same transition. After an initialization of the system in the $|-1 / 2\rangle$ state, the two arms of the interferometer are created by applying a $\pi / 2$ pulse on the $|-1 / 2\rangle \leftrightarrow|1 / 2\rangle$ second transition. Next, in order to preserve one path and to implement the quantum gate on the second, a $\pi$ pulse is applied to the $|1 / 2\rangle \leftrightarrow|3 / 2\rangle$ third transition and then the second transition is driven during a duration $\tau$. Finally, the two arms of the interferometer are merged back by applying a $\pi / 2$ pulse to the $|1 / 2\rangle \leftrightarrow|3 / 2\rangle$ second transition (Fig. 4). The sequence is completed only for pulse duration $\tau$ that ensure $a$ rotation angle $\pi(1+2 \mathrm{k})$ with $\mathrm{k}$ an integer number.

$$
\left.U_{\text {iSWAP }}^{\text {Ramsey }}=\mathbf{R}_{0}^{|1 / 2\rangle,|3 / 2\rangle}(\boldsymbol{\pi} / 2) \cdot R_{0}^{|-1 / 2\rangle,|1 / 2\rangle}(\pi+2 \mathrm{k} \pi)\right) \cdot \mathbf{R}_{0}^{|1 / 2\rangle,|3 / 2\rangle}(\boldsymbol{\pi}) \cdot \mathbf{R}_{\mathbf{0}}^{|-1 / 2\rangle, 11 / 2\rangle}(\boldsymbol{\pi} / 2)
$$

Let us now focus on the output state as a function of $\mathrm{k}$ :

$U_{i S W A P}^{\text {Ramsey }} \cdot\left(\begin{array}{l}0 \\ 1 \\ 0 \\ 0\end{array}\right)=\left(\begin{array}{c}0 \\ 0 \\ i \sin ^{2}(k \pi / 2) \\ -\cos ^{2}(k \pi / 2)\end{array}\right)$

Two cases are clearly distinguished:

if $k$ is even, corresponding to $\tau=\pi$ and an accumulated phase equal to $-i$, the output is the state $|3 / 2\rangle$ 


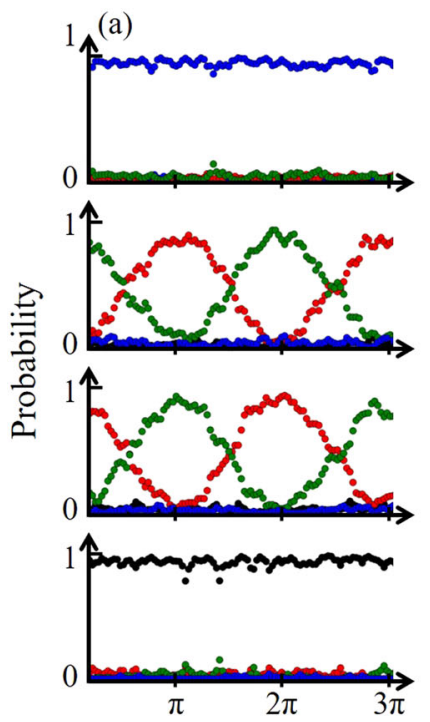

(b)

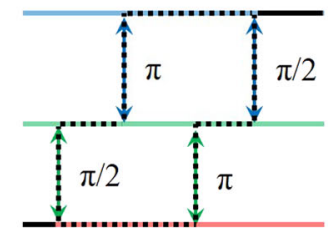

(c)
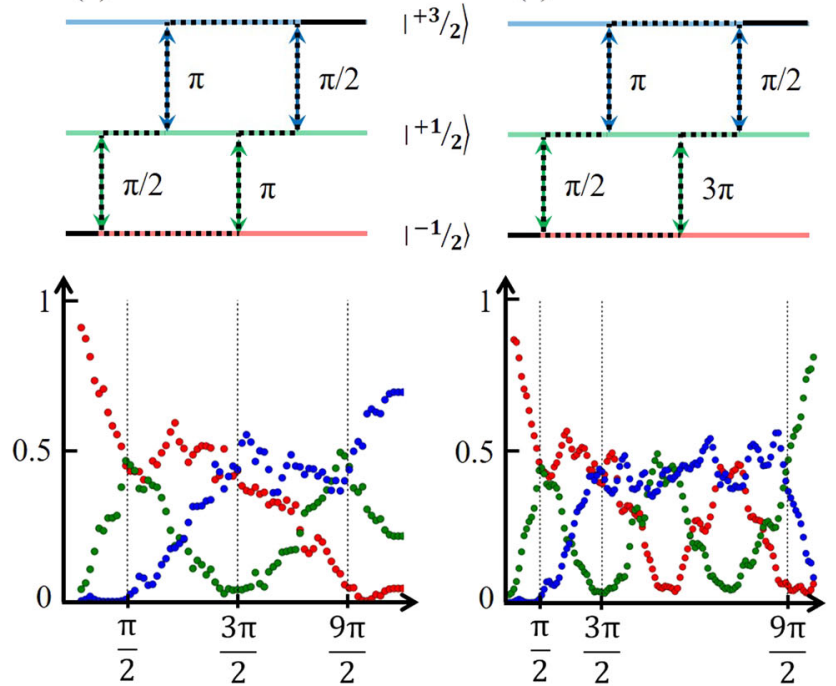

Fig. 4 iSWAP gate implementation a and phase characterization c. a A $3 \pi$ pulse on the second transition with zero phase defines an iSWAP quantum gate. Probability as a function of the pulse length of each state knowing that the initial state is $|-3 / 2\rangle,|-1 / 2\rangle,|1 / 2\rangle,|3 / 2\rangle$ from bottom to top, respectively. The states $|-3 / 2\rangle$ and $|3 / 2\rangle$ remain unchanged when the state $-|1 / 2\rangle$ and $|1 / 2\rangle$ are swapped. The $3 \pi$ rotation

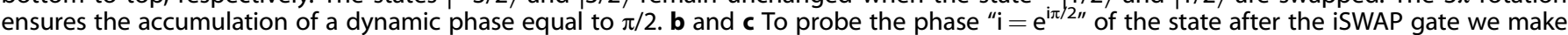
use of a three-arm Ramsey interferometry. The peculiarity of the latter is that in order to apply the quantum gate phase manipulation on only one arm of the interferometer, an additional $\pi$ pulse is considered. Consequently, the $\pi / 2$ pulses are sent on two different transitions. Probabilities $P_{p, q}$ of each state as a function of the pulse length when the initial state is $|-3 / 2\rangle,|-1 / 2\rangle,|1 / 2\rangle,|3 / 2\rangle$ from bottom to top, respectively. With this sequence, the blue state probability $P_{|-1 / 2\rangle},|3 / 2\rangle$ is maximized when the gate phase is equal to -1 and the green state probability $P_{|-1 / 2\rangle,|1 / 2\rangle}$ is maximized when the gate phase is equal to $\mathrm{i}$

if $k$ is odd, corresponding to $\tau=3 \pi$ and an accumulated phase equal to $i$, the output is the state $|1 / 2\rangle$

The evolution of the different probabilities as a function of the sequence time for a pulse equal to $\pi$ and $3 \pi$ displayed in Fig. $4 \mathrm{~b}$ and $c$, respectively provide a useful partial characterization of the gate operation. Note that to fully validate the iSWAP gate, a more complete characterization would be needed to observe this effect on the complete set of input states.

\section{Hadamard-Ramsey interferometry: coherence time}

Generally in a quantum information protocol, Ramsey fringes are used to study incoherent processes that affect a quantum superposition, thus measuring the coherence time. In the case of a qubit, at first $\pi / 2$ pulse is applied to create a coherent superposition of states, then the qubit is allowed to evolve freely in its decohering environment before being projected in the readout basis via a second $\pi / 2$ pulse. This is straightforwardly generalized to a qudit. Instead of creating a 2-state superposition using a $\pi / 2$ pulse, we apply a Hadamard gate ${ }^{40}$ that creates a multilevel coherent superposition. This method will be applied for a 3-state superposition $(|-3 / 2\rangle ;|-1 / 2\rangle$ and $|1 / 2\rangle)$. Since polychromatic pulses are used in order to drive several transitions simultaneously, the Hamiltonian of the system in the $(|-3 / 2\rangle$; $-1 / 2\rangle$ and $|1 / 2\rangle)$ basis must be dealt with in the generalized rotating frame with respect to which it takes the form ${ }^{12}$ :

$H=\frac{\hbar}{2}\left(\begin{array}{ccc}0 & \omega_{1} & 0 \\ \omega_{1} & 2 \delta_{1} & \omega_{2} \\ 0 & \omega_{2} & 2 \delta_{2}\end{array}\right)$

where $\delta_{n}$ is the pulsation detuning between the nth transition and the $v_{n}$ component of the pulse and where $\omega_{n}$ is the Rabi pulsation of the nth transition. The nuclear spin is initialized in the state $\mid-1 /$ $2\rangle$, then a first Hadamard gate is applied. It consists of a microwave pulse ensuring the same Rabi frequency for both resonances $\left(\omega=\omega_{1}=\omega_{2}\right)$ with a detuning $\delta_{1}=\omega$ of duration $\tau_{\text {Had }}=\sqrt{3} \pi / 3 \omega$ (see Supplementary information). Then the 3state superposition is allowed to evolve freely during a time $\tau$ and a second Hadamard gate is finally applied. The final state of the system after this sequence is the following:

$$
U_{\text {Had }} \cdot W_{\tau} \cdot U_{\text {Had }} \cdot\left(\begin{array}{l}
0 \\
1 \\
0
\end{array}\right)=\frac{1}{3}\left(\begin{array}{c}
e^{i \omega \tau}-1 \\
e^{i \omega \tau}+2 \\
e^{i \omega \tau}-1
\end{array}\right)
$$

where $U_{\text {Had }}$ is the Hadamard gate and $W_{\tau}$ is the free evolution of duration $\tau$. In our case, the symmetry of the Hamiltonian ensures the same dynamics for the $|-3 / 2\rangle$ and the $|1 / 2\rangle$ states, thus reducing the dimensionality of the problem: all the necessary information recurs in the dynamic of the $|-1 / 2\rangle$ state, consisting in oscillations of the Rabi pulsation $\omega$ in between the state $|-1 / 2\rangle$ and the two states $|1 / 2\rangle$ and $|-3 / 2\rangle$ (Fig. $5 d$ ). The oscillation of the $|-1 / 2\rangle$ state is displayed in Fig. $5 b$ and c, respectively, for a waiting time below $1 \mu \mathrm{s}$ and above $25 \mu \mathrm{s}$. As with a qubit, incoherent interactions with the environment break the phase coherence causing a damping of the oscillation amplitudes (Fig. 5e). The characteristic time of this damping is in the order of $90 \mu \mathrm{s}$ for this single nuclear spin qudit $(\mathrm{d}=3)$. This is smaller than a phase coherence time of two-state superposition, of the order of $300 \mu \mathrm{s}$ on this system. ${ }^{12} \mathrm{~A}$ deeper analysis is needed to explain this difference. The method can be applied to any qudit system.

\section{DISCUSSION}

For a single nuclear spin qudit, we have investigated different interferometric protocols to measure the geometric phase, quantum gate phase and finally the multilevel quantum superposition coherence time. The periodicities of geometric phase accumulation, related to intrinsic properties of the Hilbert space under investigation, have been validated using Ramsey fringes. Through an analogy in between a qudit and 2-qubits, we implemented an iSWAP gate. The phase of this gate can directly be measured using multi-transition Ramsey fringes. Finally, we 
(a)

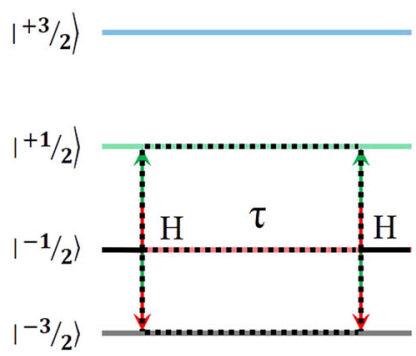

(b)

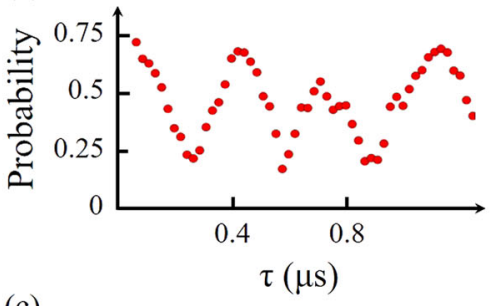

(c)

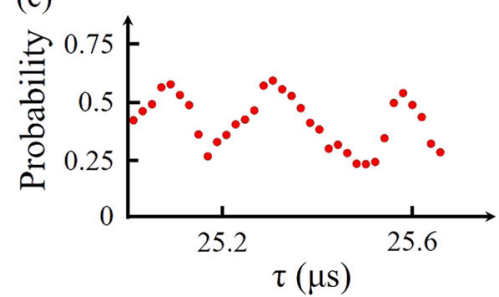

(d)

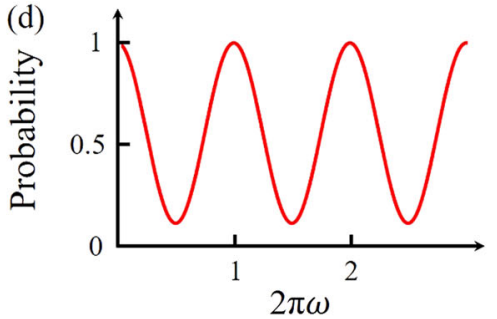

(e)

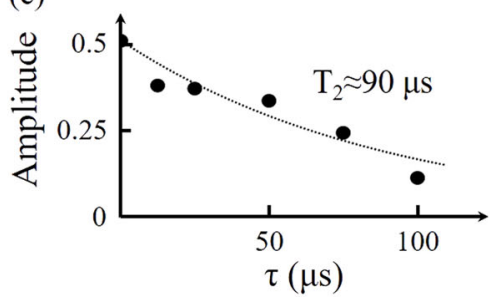

Fig. 5 3-State coherence time. a Pulse sequence to measure a multi-state superposition. A first Hadamard gate creates a coherent superposition, then the system evolves freely during a time $\tau$. Finally, a second Hadamard gate is sent to close the interferometric path. $\mathbf{b}$ Experimental oscillations of the red state probability as a function of the free evolution time. $\mathbf{c}$ The oscillations are still visible for $\tau$ of the order of $25 \mu \mathrm{s}$. d Theoretical evolution of the red state probability $P_{|-1 / 2\rangle,|-1 / 2\rangle}$ after applying two Hadamard gates separated in time by a free evolution time $\tau$ revealing a good agreement with the measurement. e Damping of the amplitude exhibits a coherence time for the 3-states superposition of the order of $90 \mu \mathrm{s}$

have been able to measure the coherence time of a 3-states superposition of the single nuclear spin through a protocol using two Hadamard gates generalizing the coherence time measurement of a two-level system to any n-level system. These measurements highlight the potentialities of molecular magnets for quantum information processing. The next challenge towards this latter objective is to fabricate scalable molecular magnet qudits architectures. One direction is the use of multi-magneticcentre molecular magnet. ${ }^{41}$ Another concerns molecular units in hybrid architectures. Ensembles of molecular magnets have been used so far to enhance the collective coupling with microwave photons at very low temperature ${ }^{42}$ as well as temperature up to $50 \mathrm{~K}$ using high $T_{c}$ superconductive microwave planar resonator. ${ }^{43}$ The next objective is to coherently couple a single molecular spin to a single photon, as already achieved using spin quantum dots. $^{44,45}$

\section{METHODS}

The set-up is the same as that detailed $i^{25}$ except the microwave pulse generation. In this experiment, the microwave pulses were generated with a Tektronix 24 Giga sampling Arbitrary Wave Generator.

\section{DATA AVAILABILITY}

The data supporting the findings of this study are available by the corresponding author upon request.

\section{ACKNOWLEDGEMENTS}

We gratefully acknowledge E. Eyraud, D. Lepoittevin, and C. Hoarau for their technical contributions and motivating discussions. We thank J.F. Motte, T. Crozes, B. Fernandez, S. Dufresnes and G. Julie for Nano-fabrication development, S. Thiele and $C$. Thirion for help with the development of the experiment. Samples were fabricated in the NANOFAB facility of the Néel Institute. This work is partially supported by the French National Agency of Research through the ANR-13- BS100001 MolQuSpin, by the German Research Foundation (DFG) through the Transregio Project No. TR88 "3MET", by the Alexander von Humboldt Foundation, and the ERC advanced grant MoQuOS n. 741276.

\section{AUTHOR CONTRIBUTIONS}

C.G., E.B., W.W. and F.B. designed, carried out and analysed the experiments, S.K and M.R. designed and synthesized the molecule; C.G. and R.B wrote the paper, which was revised by all authors.

\section{ADDITIONAL INFORMATION}

Supplementary information accompanies the paper on the npj Quantum Information website (https://doi.org/10.1038/s41534-018-0101-3).

Competing interests: The authors declare no competing Interests.

Publisher's note: Springer Nature remains neutral with regard to jurisdictional claims in published maps and institutional affiliations.

\section{REFERENCES}

1. Feynman, R. Simulating physics with computers. Int. J. Theor. Phys. 21, 467-488 (1982).

2. Ladd, T. et al. Quantum computers. Nature 464, 45-53 (2010).

3. Nielsen, M., Chuang, I. \& Grover, L. Quantum computation and quantum information. Am. J. Phys. 70, 558-559 (2002).

4. Ashok, M. \& Stroud, C. R. Jr. Multivalued logic gates for quantum computation. Phys. Rev. A 62, 052309 (2000).

5. Bullock, S. S., O'Leary, D. P. \& Brennen, G. K. Asymptotically optimal quantum circuits for d-level systems. Phys. Rev. Lett. 94, 230502 (2005).

6. Kues, M. et al. On-chip generation of high-dimensional entangled quantum states and their coherent control. Nature 546, 622 (2017).

7. Kiktenko, E., Fedorov, A., Strakhov, A. \& Man'ko, V. Single qudit realization of the Deutsch algorithm using superconducting many-level quantum circuits. Phys. Lett. A 379, 1409-1413 (2015).

8. Farhi, E. \& Gutmann, S. Analog analogue of a digital quantum computation. Phys. Rev. A 57, 2403-2406 (1998).

9. Kessel', A. \& Ermakov, V. Multiqubit spin. J. Exp. Theor. Phys. Lett. 70, 61-65 (1999).

10. Lapkiewicz, R. et al. Experimental non-classicality of an indivisible quantum system. Nature 474, 490-493 (2011).

11. Lanyon, B. et al. Simplifying quantum logic using higher-dimensional Hilbert spaces. Nat. Phys. 5, 134-140 (2008).

12. Godfrin, C. et al. Operating quantum states in single magneticmolecules: implementation of Grover's quantum algorithm. Phys. Rev. Lett. 119, 187702 (2017).

13. Leuenberger, M. \& Loss, D. Quantum computing in molecular magnets. Nature 410, 789-793 (2001).

14. Jenkins, M. D. et al. Coherent manipulation of three-qubit states in a molecular single-ion magnet. Phys. Rev. B 95, 064423 (2017). 
15. Sohbi, A., Zaquine, I., Diamanti, E. \& Markham, D. Logical and inequality-based contextuality for qudits. Phys. Rev. A 94, 032114 (2016)

16. Wilczek, F. \& Zee, A. Appearance of gauge structure in simple dynamical systems Phys. Rev. Lett. 52, 2111 (1984).

17. Malik, M. et al. Multi-photon entanglement in high dimensions. Nat. Photonics 10 248 (2016)

18. Wheeler, A. \& Zurek, W. H. Quantum theory of measurement. (Princeton University Press, Princeton, 1983).

19. Brune, M. et al. Observing the progressive decoherence of the "meter" in a quantum measurement. Phys. Rev. Lett. 77, 4887 (1996).

20. Pirandola, S., Mancini, S., Braunstein, S. L. \& Vitali, D. Minimal qudit code for a qubit in the phase-damping channel. Phys. Rev. A 77(3), 032309 (2008).

21. Ramsey, N. F. Molecular Beams. (Oxford University Press, New York, 1985).

22. Young, T. The Bakerian Lecture: on the mechanism of the eye. Philos. Trans. R. Soc. Lond. 91, 23-88 (1801).

23. Carnal, O. \& Mlynek, J. Young's double-slit experiment with atoms: a simple atom interferometer. Phys. Rev. Lett. 66, 2689 (1991).

24. Vincent, R., Klyatskaya, S., Ruben, M., Wernsdorfer, W. \& Balestro, F. Electronic read-out of a single nuclear spin using a molecular spin transistor. Nature $\mathbf{4 8 8}$ 357-360 (2012).

25. Thiele, S. et al. Electrically driven nuclear spin resonance in single-molecule magnets. Science 344, 1135-1138 (2014)

26. Shiddiq, M. et al. Enhancing coherence in molecular spin qubits via atomic clock transitions. Nature 531, 348-351 (2016).

27. Ishikawa, N., Sugita, M. \& Wernsdorfer, W. Quantum tunneling of magnetization in lanthanide single-molecule magnets: Bis(phthalocyaninato)terbium and Bis (phthalocyaninato)dysprosium Anions. Angew. Chem. 117, 2991-2995 (2005).

28. Godfrin, $C$. et al. Electrical read-out of a single spin using an exchange-coupled quantum dot. ACS nano 11, 3984-3989 (2017).

29. Pancharatnam, S. Generalized theory of interference, and its applications. Proc. Indian Acad. Sci. 44, 247-262 (1956).

30. Berry, M. Quantal phase factors accompanying adiabatic changes. Proc. R. Soc. A: Math., Phys. Eng. Sci. 392, 45-57 (1984).

31. Simon, B. Holonomy, the quantum adiabatic theorem and Berry's phase. Phys. Rev. Lett. 51, 2167 (1983).

32. Samuel, J. \& Bhandari, R. General setting for Berry's phase. Phys. Rev. Lett. 60, 2339 (1988)

33. De Chiara, G. \& Palma, G. Berry phase for a Spin $1 / 2$ particle in a classical fluctuating field. Phys. Rev. Lett. 91, 090404 (2003).

34. Yale, C. et al. Optical manipulation of the Berry phase in a solid-state spin qubit. Nat. Photonics 10, 184-189 (2016).
35. Berger, S. et al. Exploring the effect of noise on the Berry phase. Phys. Rev. A 87 060303 (2013).

36. Wernsdorfer, W. \& Sessoli, R. Quantum phase interference and parity effects in magnetic molecular clusters. Science 284, 133-135 (1999).

37. Leek, P. et al. Observation of Berry's phase in a solid-state qubit. Science $\mathbf{3 1 8}$, 1889-1892 (2007).

38. Neeley, M. et al. Emulation of a quantum spin with a superconducting phase qudit. Science 325, 722-725 (2009).

39. Man'ko, M. \& Man'ko, V. Entanglement and other quantum correlations of a single qudit state. Int. J. Quantum Inf. 12, 1560006 (2014).

40. Leuenberger, M. \& Loss, D. Grover algorithm for large nuclear spins in semiconductors. Phys. Rev. B 68, 165317 (2003).

41. Moreno Pineda, E., Lan, Y., Fuhr, O., Wernsdorfer, W. \& Ruben, M. Exchange-bias quantum tunnelling in a $\mathrm{CO}_{2}$-based $\mathrm{Dy}_{4}$-single molecule magnet. Chem. Sci. 8 1178-1185 (2017).

42. Mergenthaler, M. et al. Strong coupling of microwave photons to antiferromagnetic fluctuations in an organic magnet. Phys. Rev. Lett. 119, 147701 (2017).

43. Bonizzoni, C., Ghirri, A. \& Affronte, M. Coherent coupling of molecular spins with microwave photons in planar superconducting resonators. Adv. Phys. X, 1435305 3 :1. (2018).

44. Landig, A. J. et al. Coherent spin-photon coupling using a resonant exchange qubit. Nature 560, 179 (2018).

45. Samkharadze, N. et al. Strong spin-photon coupling in silicon. Science 359, 1123-1127 (2018).

Open Access This article is licensed under a Creative Commons Attribution 4.0 International License, which permits use, sharing, adaptation, distribution and reproduction in any medium or format, as long as you give appropriate credit to the original author(s) and the source, provide a link to the Creative Commons license, and indicate if changes were made. The images or other third party material in this article are included in the article's Creative Commons license, unless indicated otherwise in a credit line to the material. If material is not included in the article's Creative Commons license and your intended use is not permitted by statutory regulation or exceeds the permitted use, you will need to obtain permission directly from the copyright holder. To view a copy of this license, visit http://creativecommons. org/licenses/by/4.0/.

(c) The Author(s) 2018 